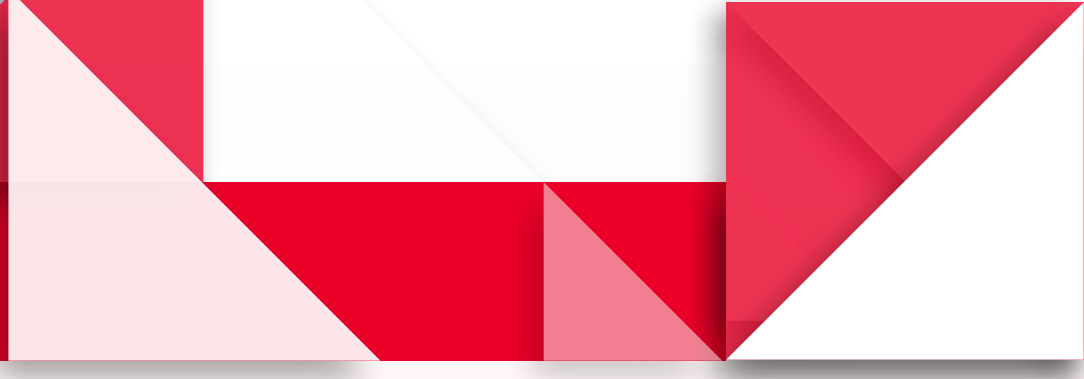

\title{
El modelo de resolución de 3 problemas en una perspectiva de investigación como práctica social normada
}

Eliseo Ramírez Rincón ${ }^{1}$

- The model of problem solving in a perspective of research as a regulated social practice

- O modelo de resolução de problemas em uma perspectiva de investigação como prática social regulada

\footnotetext{
Resumen

"El profesor de matemáticas no debería contentarse con dispensar el saber, sino que también debería intentar desarrollar en los estudiantes la capacidad de usar ese saber; debería insistir en el saber hacer, en las actitudes adecuadas, en los hábitos intelectuales deseables". Polya (1979).

Este artículo no está interesado en la discusión sobre problema y resolución de problemas, sino en revisar la naturaleza y coherencia de la metodología establecida por la investigación a través del modelo de resolución de problemas desde la perspectiva de investigación (MRPPI), desde un marco comparativo con la ingeniería didáctica, que como metodología de investigación, tiene unos desarrollos reconocidos por diversas culturas y áreas del conocimiento. Además, interesa determinar si la metodología al interior del grupo Probleduciencias, adscrito a dicho modelo, determina una práctica social normada, en la postura de la socioepistemología (Cantoral, Farfán, Lezama, Martínez, 2006). Este, surge del trabajo doctoral del autor en el grupo de Probleduciencias.
}

Palabras clave:

didáctica matemática, Probleduciencias, práctica social normada, reconocimiento del sujeto. 


\section{Abstract}

"Mathematics teachers should not be content with dispensing knowledge, they should also try to develop the ability to use this knowledge in their students" they should insist on knowing to do and enhancing attitudes and intellectual habits" (Polya, 1979).

This article is not interested in the discussion of problem and problems solving, but in reviewing the nature and consistency of the methodology established by the research through the model of problems solving from the perspective of research (MRPPI), from a comparative framework within the Didactic engineering, which has some advances recognized by various cultures and fields of knowledge as a research methodology. In addition, it is important to determine if the methodology to the inside of the Group Probleduciencias, attached to the model, determines a regulated social practice based on epistemology (Cantoral, Farfan, Lezama, Martinez, 2006).

\section{Resumo}

"O professor das matemáticas não deveria se contentar em distribuir o saber, mas também deveria tentar desenvolver nos estudantes a capacidade de usar esse saber; deveria insistir no saber fazer, na atitudes adequadas, nos hábitos intelectuais desejáveis" (Polya, 1979, tradução livre).

Este artigo não está inserido na discussão sobre problema e resolução de problemas, mas em revisar a natureza e coerência da metodologia estabelecida pela pesquisa por meio do Modelo de Resolução de Problemas a partir da Perspectiva de Investigação, a partir de uma marco comparativo com a Engenharia Didática que, como metodologia de pesquisa, possui alguns desenvolvimentos reconhecimentos por diversas culturas e áreas do conhecimento.

Além disso, interessa determinar se a metodologia no âmbito do grupo Probleduciencias, ligado ao Modelo, determina uma prática social regulada, na postura da Socioepistemologia (Cantoral, Farfán, Lezama, Martínez, 2006). Este resulta do trabalho de doutorado do autor no grupo Probleduciencias.

\section{Keywords:}

mathematics

Didactics, regulated social practice, subject recognition.

\section{Palavras-chave:} didáctica da Matemática; Prática Social Regulada; Reconhecimento do Sujeito. 


\section{Introducción}

Dado que el modelo de resolución de problemas desde la perspectiva de investigación (MRPPI), es el referente teórico-metodológico que articula al grupo de investigación Probleduciencias, desde un enfoque sistémico con el plano de lo organizativo (gráfico 3) y con los procesos (gráfico 4) en niveles y relaciones de complejidad diferenciados en lo personal, cultural y social que dan cuenta de la integralidad de la persona que se está formando y del saber situado con el que lo hace. Este saber situado, correspondió a la matemática escolar, desde la cual se configuró y realizó la tesis doctoral "Enseñanza de la función derivada con el uso de infinitesimales como alternativa para reducir los conflictos semióticos de los estudiantes" (Ramírez, 2012), cuyo foco de interés fue el lenguaje matemático del cálculo, desde su rigor y formalismo.

En este artículo se hará énfasis en generalizar el MRPPI como metodología de investigación y como práctica social normada, porque en la fase exploratoria y en el marco teórico de la tesis, se encontraron estos aspectos que permitieron dar sentido a la cultura en la que la tríada profesor/estudiante/saber interactuó en el trabajo investigativo de la tesis.

En el MRPPI el plano de lo organizativo está integrado por los nodos de planeación y reconocimiento del sujeto. Este se relaciona con el saber situado desde las dimensiones del ser, los contextos, el ambiente de aprendizaje y los procesos que se desarrollan con la educación (gráfico 4). El modelo también concibe así la integralidad del ser desde los nodos (gráficos 1 y 2) y los procesos de la educación que al ser articulados intencionalmente (estudiante, profesor, institución educativa, familia, entorno, saber) evidencian niveles significativos en el desarrollo integral humano, como una práctica social normada (gráficos 3 y 4), al planear acti- vidades intencionadas desde el reconocimiento de las dimensiones del sujeto, del contexto en el que lo hace y del saber situado.

La consideración de que el MRPPI genera una práctica social normada se sustenta en la tesis de la socioepistemología (Cantoral et al., 2006) definida como: "la actividad humana como base de la construcción de los sistemas conceptuales, no se limita a caracterizar lo que el ser humano hace, sino a problematizar las causas del porqué lo hace... ¿ Qué me (nos) hace hacer lo que hago (hacemos)...?" (p. 85).

Por consiguiente, se quiere indagar en cómo se normalizaron estas prácticas en el modelo, si fueron producto de una construcción colectiva para responder al trabajo de investigación del grupo, si corresponde a otro aspecto metodológico o a una combinación de estos. En ese sentido se recurrirá a la ingeniería didáctica (Artigué, 1995) como metodología de investigación, con el fin de estudiar el carácter metodológico del Modelo.

\section{Discusión}

En el grupo Probleduciencias se propone una misión y una visión que marcan la ruta y el compromiso social que se adquiere en la educación como proceso, con el fin de dar sentido a la integralidad del ser humano.

\section{Misión del grupo}

Genera conocimiento como resultado de la investigación en su campo de interacción, en diferentes niveles educativos y con participación de profesionales y estudiantes de diversas disciplinas involucrados en la formación en ciencias, matemáticas, ingenierías y otros campos del saber.

Asume el compromiso de contribuir al desarrollo humano integral mediante la investigación y la práctica educativa. 
Incide en procesos de formación, investigación y de proyección social en contextos específicos.

Contribuye a la generación de políticas públicas.

\section{Visión del grupo}

Será reconocido como un grupo generador de conocimiento educativo y pedagógico en el campo de la educación la calidad de vida y el desarrollo humano, en la perspectiva de una vida digna para todos.

Como resultado de su producción establecerá alianzas con grupos de investigación y redes nacionales e internacionales para la realización de investigaciones relevantes para el país, la región latinoamericana y caribeña.

Participa en la formación doctoral a nivel internacional.

A partir de la misión y la visión, se generan también un objetivo y una práctica pedagógica que propone lineamentos específicos de trabajo, dentro de la formación de los profesores de ciencias a nivel de pregrado y posgrado.

\section{Objetivo del grupo Probleduciencias del MRPPI}

Corresponde a una mirada desde la didáctica de las ciencias, con el fin de producir conocimiento en este campo específico y contribuir a la consolidación de una postura teórica unificada pero flexible en el ámbito de la enseñanza de las ciencias en carreras de ciencias naturales y afines, matemáticas e ingenierías (hoy en día otras disciplinas) a través de la educación centrada en la resolución de problemas, a partir del modelo "resolución de problemas en una perspectiva de investigación" desarrollado y en consolidación en el grupo del doctorado.

\section{Práctica pedagógica. Objetivos}

Contribuir a la formación de docentes a través de la interacción educativa y la investigación formativa, encaminadas a proponer estrategias de solución a problemáticas de calidad de vida, desarrollo humano y educación en contexto.

Desarrollar estrategias de formación investigativa relacionada con la interacción pedagógica y didáctica en el campo, de las ciencias y otros campos del saber, de manera que se generen alternativas de enseñanza encaminadas a propiciar aprendizajes con sentido y procesos de autorregulación en los profesores en formación (pregrado y posgrado).

\section{Práctica pedagógica. Campos de acción}

El grupo interactúa a nivel nacional e internacional con instituciones de educación formal en los niveles básico, medio y superior; educación no formal. Tal es el caso del Jardín Botánico, ONG, convenios de cooperación con Unesco y 
otros organismos intergubernamentales, OEA, ministerios de Educación y de la Protección Social, Secretaría Distrital de Ambiente y de Educación, entre otros.

Teniendo en cuenta que el grupo está articulado al MRPPI, el cual determina sus pautas en los roles teórico, metodológico, pedagógico y didáctico, y de acuerdo con el carácter del mismo respecto a la consideración de integralidad del ser desde la educación como proceso, porque: se la concibe como un proceso que contribuye a la formación de las personas y les permite asumir una concepción del mundo, a partir de una preparación político-ideológica, intelectual, ética y moral, estética y lúdica, física, comunicativa y en el afecto. (Jessup y Pulido, 1998, p. 112 y 115).

De acuerdo con esta consideración procesual de la integralidad, se contempla en el MRPPI el favorecimiento de:

La planificación y el control de acciones por parte de los estudiantes, como parte del proceso de aborde de la situación problema, la concreción del problema y el establecimiento de pautas encaminadas a su resolución.
El establecimiento de redes de relaciones conceptuales entre saberes propios de la temática y articulaciones conceptuales con otros saberes.

De otra parte, los estudiantes participan activamente en la propuesta investigativa a través de la identificación de variables, el planteamiento del problema, en la experimentación o desarrollo y en las conclusiones o análisis a posteriori de las situaciones problema. Además, MRPPI tiene en cuenta que la planeación del trabajo se centra en el proceso de aprendizaje organizado a partir de los principios teóricos y metodológicos, los cambios de actitudes y valores, la apropiación de la epistemología científica, el proceso de resolución de problemas (RPPI) significativos para los estudiantes; porque, al tener en cuenta los intereses de los estudiantes, no hay imposiciones sino acuerdos de trabajo con ellos.

Lo anterior se puede interpretar a través de las relaciones que se establecen en el nodo planeación (gráfico 1). Estas son tenidas en cuenta en la identificación, propuesta y análisis a priori de la situación problema en cuestión.

Gráfico 1. Nodo de planeación

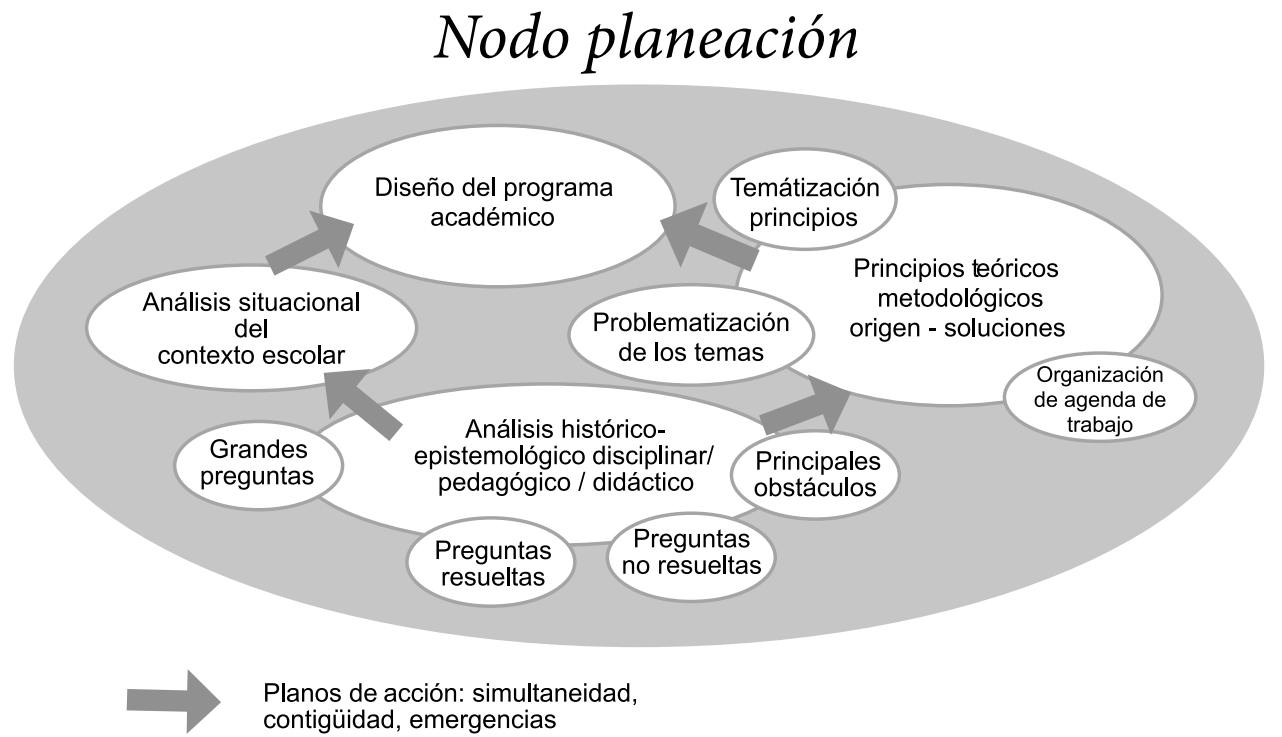


El nodo planeación está articulado con el de reconocimiento del sujeto (gráfico 2) porque hay interés en quién aprende, qué aprende y cómo aprende; por ello se contemplan algunas dimensiones enmarcadas en el nodo del gráfico 2 , las cuales incluyen aspectos tanto personales (ideas previas, intereses) como comunitarios (entorno, necesidades); lo que permite al estudiante hacer aportes a la situación problema que investiga a su propio ritmo y a la vez construir su propio saber a partir de los avances alcanzados en ella, como un saber situado.

Gráfico 2. Nodo de reconocimiento del sujeto

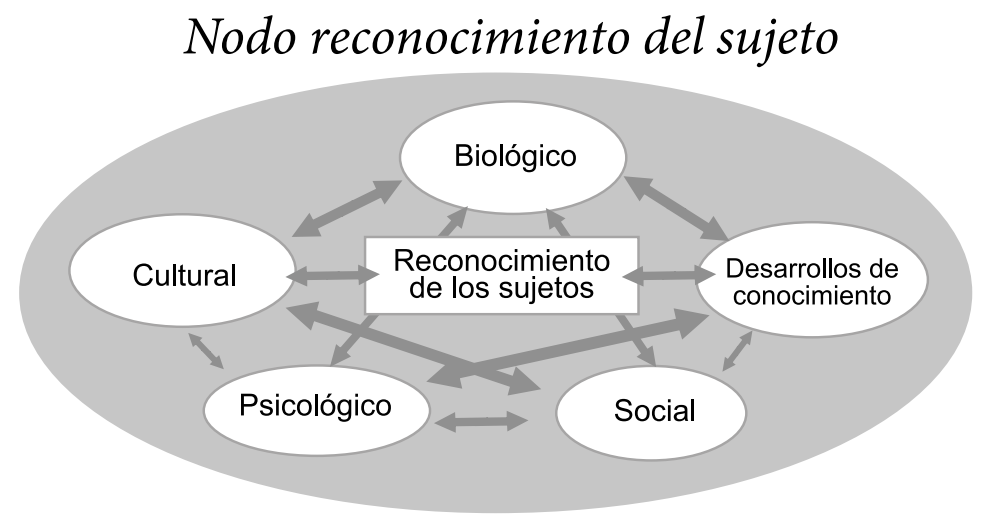

El gráfico 3 presenta el plano organizativo del modelo a través del cual se planifican, diseñan y discuten las propuestas de investigación teniendo en cuenta el instrumento que se deriva de este.

\section{Gráfico 3. Plano de lo organizativo}

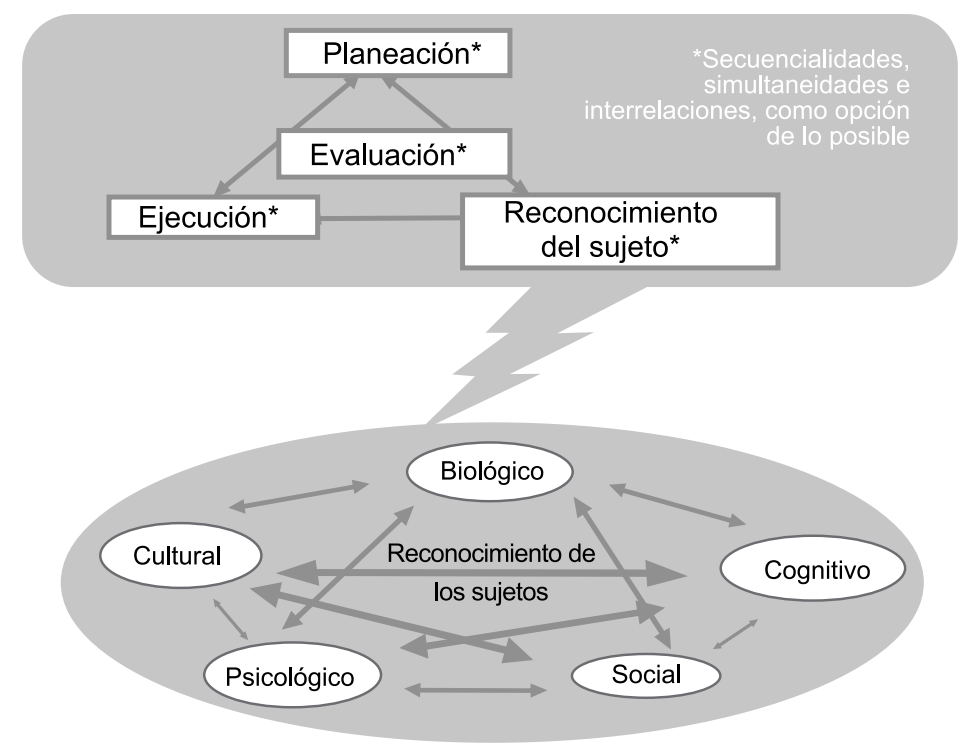

El plano de lo organizativo, hace referencia a la forma como en el MRPPI se concibe al sujeto, desde las dimensiones: cultural (entorno inmediato, local), social (entorno global), biológico (genotipo-fenotipo), cognitiva (tiene en cuenta los procesos de atención, interacción, percepción, memoria, representación, 
comunicación) y psicológica (alteraciones de las emociones, qué las produce y los efectos en el sujeto) (Jessup, 1998; Pulido, 2007).

En consecuencia, en el proceso de planeación del profesor para la generación de aprendizajes, es pertinente asumir la propuesta del MRPPI (Jessup, 2004) para la enseñanza de las ciencias naturales, matemáticas, ingenierías, etc., acorde con las particularidades de la acción educativa en cada una de ellas, como parte de la formación integral de los estudiantes. Las estrategias adoptadas por el docente influyen en las transformaciones cognitivas y actitudinales de los estudiantes, además porque estas están ligadas al problema, seguimiento y asesoría que se desarrolle durante el proceso de resolver la situación problémica planteada, diseñada y por resolver que incentiva el interés de los estudiantes para el desarrollo de explicaciones científicas o de otra naturaleza relacionadas con el mundo. Para tal efecto, el modelo asume la resolución de problemas como:
(...) un proceso mediante el cual, una persona que se enfrenta a un problema, trata de identificarlo, de delimitarlo, de explorar posibilidades de resolverlo, de elegir las estrategias adecuadas para lograrlo a partir de sus desarrollos individuales, de llevarlas a la práctica mediante la aplicación de métodos y técnicas apropiados y de obtener cierta aproximación a la solución del mismo. (Jessup y Pulido, 2001, p. 302-305).

Dado que la resolución de problemas es entendida por el modelo como un proceso que debe contemplar acciones en la educación de un estudiante para formarse integralmente; en este sentido, los problemas que se plantean no son disciplinares, sino que se les considera generadores de aprendizajes dentro del marco de educación contemplado en la complejidad del modelo, según las relaciones establecidas en el gráfico 4.

Gráfico 4. Procesos involucrados en el desarrollo integral de las personas

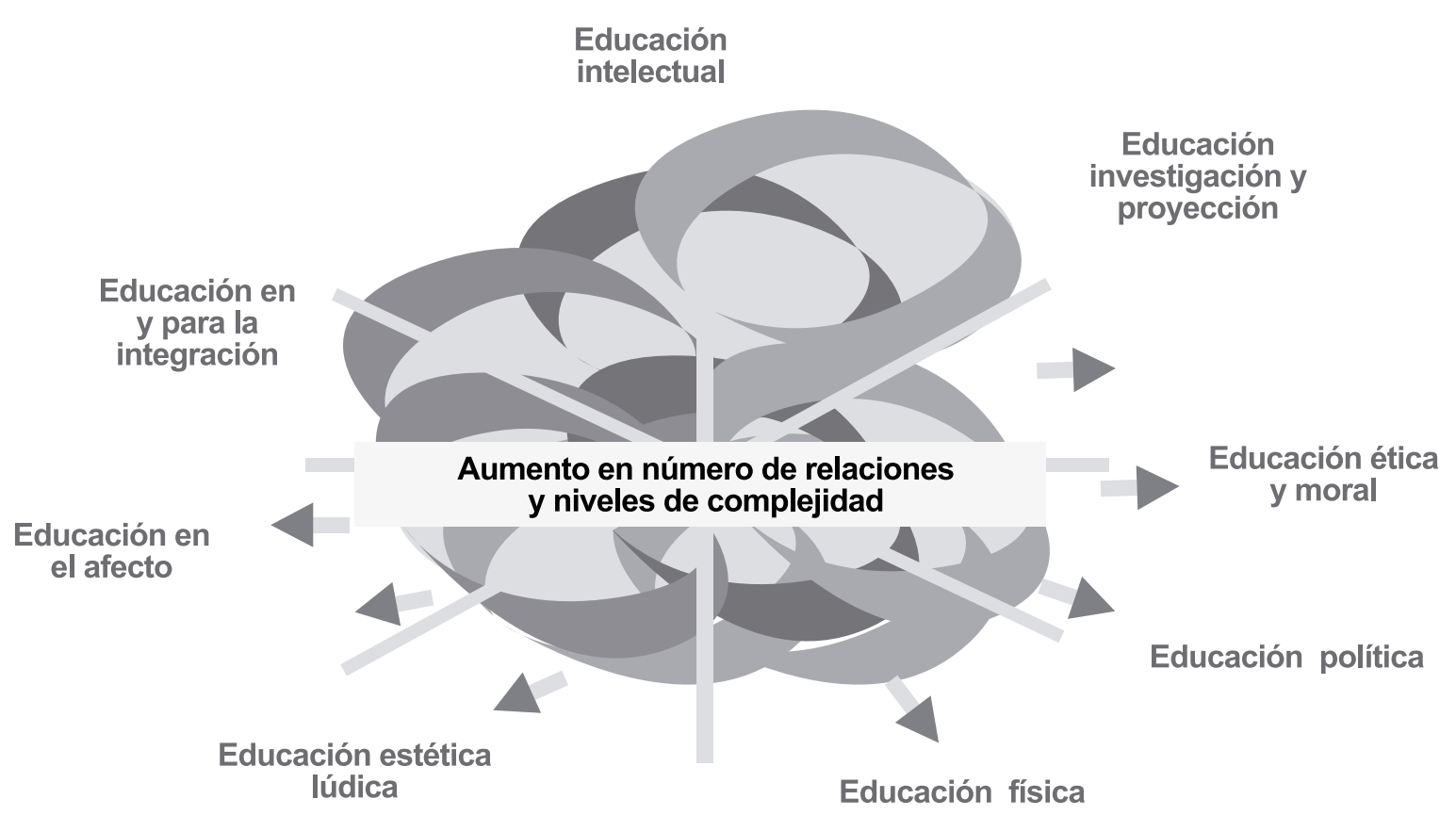


El gráfico 4 presenta la forma como se establecen las relaciones de un ser humano en proceso de formación permanente en y para la vida. Llama la atención en que la educación es la que permite trascender el desarrollo del ser desde la complejidad y lo sistémico. Estas relaciones tienen en cuenta al sujeto desde las dimensiones: cognitiva, social/cultural, didáctica, epistemológica y psicológica; que conforman el plano de lo organizativo (gráfico 3) (Jessup, 1998; Pulido, 2007).

A partir de la información articulada en las relaciones que se establecen en y entre los gráficos 1, 2, 3 y 4, se hace evidente entonces establecer cómo se proponen, organizan, llevan a la práctica y evalúan las actividades según el modelo. En este sentido el instrumento (anexo 1), generado en el plano de lo organizativo, permite fijar en detalle la planeación y desarrollo y resultados de la investigación.

Dado que el MRPPI es flexible, sistémico y dinámico, susceptible de ser desarrollado con diferentes metodologías, se le hará una ingeniería didáctica (ID) con el fin de retroalimentar y autorregular los procesos propios del modelo.

\section{La ingeniería didáctica (ID)}

Se inicia en Francia, en la década de 1960, en los trabajos desarrollados en los Institutos de Investigación en Enseñanza de las Matemáticas (IREM), que intentaban contrarrestar los efectos nocivos de la propuesta del grupo Bourbaki, sobre la enseñanza axiomatizada de las matemáticas en el nivel básico. Esta problemática derivó de una parte en la comprensión, mediante el estudio sistematizado de Piaget, de los procesos cognitivos propios de los niños y de otra, en la capacitación de los profesores de educación básica sobre contenidos propiamente matemáticos, nuevos o ya olvidados (Douady, 1995).

\section{La ID estudia dos aspectos}

Las relaciones entre la investigación y la acción en el sistema de enseñanza, deben generar una dinámica en la experimentación basada en las realizaciones didácticas en el aula, que contemplan la concepción, realización, observación y análisis de secuencias de enseñanza.

En este aspecto el modelo ha construido una metodología cuyo centro de atención es la educación de la persona, reafirmando y diferenciando las dimensiones, y las diversas relaciones entre ellas, para ello ha dispuesto un plano de lo organizativo desde el que se establecen las relaciones en las dimensiones que en un trabajo particular se deben privilegiar de acuerdo con los objetivos y las necesidades propias de una comunidad específica. El plano de lo organizativo se apoya en un instrumento que permite planear, presentar, aplicar y evaluar las acciones de una práctica social normada.

El papel de las prácticas investigativas en clase, orientado por el registro 
basada en la confrontación entre el análisis a priori y a posteriori, a través de la triangulación Estudiante-profesor-investigador y de éstos a su vez con un investigador externo, evidencian una ruta normada por las acciones y prácticas del quehacer académico e investigativo que la convierten en una "práctica social normada"..

El modelo asume este rol con los estudios de caso y en el plano de lo organizativo se contempla a través del instrumento con el cual se planea y se evalúa un trabajo determinado, confrontando como análisis a priori y a posteriori la información registrada en la planeación, experimentación y resultados del estudio; ya sea de caso o grupal, porque en el modelo cada paso enmarcado en el plano organizativo debe ser sometido a confrontación tanto interna (aula, profesor, estudiantes, investigador) como externa (investigador externo, validación del instrumento y de los resultados).

La ID se interesa tanto en las propuestas pedagógicas basadas en investigación como en las metodologías específicas de la misma investigación. Esto permite que ella pueda ser vista en sí como una metodología de investigación dado que presenta como características el procurar un sistema experimental basado en las realizaciones didácticas en clase; el llevar un registro de estudios de caso cuya validación se basa en la confrontación del análisis a priori y a posteriori, y cuyos objetivos pueden ser diversos (Artigue, Douady, Moreno y Gómez, 1995; Farfán 1997). Este aspecto es el que permite evaluar el modelo desde una perspectiva diferente a la naturaleza del mismo.

La ID según Artigue, distingue tres dimensiones:

- Dimensión epistemológica: asociada a las características del saber puesto en funcionamiento, en este caso de la función derivada y su lenguaje en la resolución de problemas.
- Dimensión cognitiva: asociada a las características cognitivas de los alumnos a los que se dirige la enseñanza, es decir a la persona que aprende, vista en sus dimensiones (ética, social, psicológica).

- Dimensión didáctica: asociada a las características del funcionamiento del sistema de enseñanza. En este sentido propone una alternativa que favorezca el proceso de enseñanza/aprendizaje de un saber particular de las ciencias, matemáticas o ingenierías.

En el plano de lo organizativo, el modelo contempla estas dimensiones y además asume la social-cultural, porque individualiza a las personas desde la pluriculturalidad nacional.

La investigación basada en la ID presenta cuatro fases (Artigue et al., 1995), que para efectos de este artículo se reconocen así:

- Planeación: en la que se contempla el análisis de cuestiones epistemológicas, curriculares, contextuales en el ámbito social, escolar, económico, institucional, etc. Con el fin de determinar el objetivo de la investigación o del trabajo en el aula, se realiza a través del instrumento que determina el plano de lo organizativo (gráfico 3).

- Diseño de las situaciones didácticas: se pretende obtener una visión a priori del quehacer del alumno, en el desarrollo de tales situaciones y sobre las posibles variables que pueden intervenir en el proceso; de esta manera se pretende prevenir los posibles comportamientos de los sujetos que intervienen en el proceso. A partir del plano de lo organizativo se diseñan las situaciones, dependiendo de la actividad a realizar planeada en el instrumento. 
- Desarrollo o experimentación: se pone en juego lo planeado en las dos fases anteriores y se procura observar y detallar el proceso educativo de la mejor manera posible.

- Evaluación: se realiza un análisis entre lo planeado y lo obtenido con el fin de encontrar la forma de validar la investigación en sí.

En el modelo se contemplan estas fases en el plano de lo organizativo a través del instrumento que le es propio. Cualquier actividad debe ser planeada, discutida, diseñada, aplicada o experimentada y evaluada. El modelo se interesa por establecer la dinámica del saber en el aula y fuera de ella, en relación con los estudiantes. En este sentido sería pertinente que en el modelo se revisara la posibilidad de estudiar el conocimiento y el saber contextualizados en las prácticas sociales normadas, por considerar que son estas las que dan sentido a cualquier conocimiento o saber y no al contrario como lo establece la socioepistemología en sus innumerables investigaciones y trabajos reportados en el mundo.

\section{La socioepistemología}

La actividad humana como base de la construcción de los sistemas conceptuales no se limita a caracterizar lo que el ser humano hace, sino a problematizar las causas del porqué lo hace... ¿̇Qué me (nos) hace hacer lo que hago (hacemos), así mismo respecto a la construcción, adquisición y difusión del saber matemático, llama la atención por el hecho de que la actividad humana es la que crea al objeto matemático, a través de prácticas sociales normadas; en las que el énfasis no está en el objeto ni en su representación, sino en la modelación de la práctica social en la construcción de conocimiento matemático. Las prácticas sociales, entendidas no como lo que hace en sí el individuo o el grupo, sino aquello que les hace hacer lo que hacen, en términos de Covián (2005, citado por Cantoral et al., 2006, p. 85).

\section{Conclusiones}

El MRPPI, está construyendo su marco teórico; para este se contemplan definiciones, metodología y evaluación de las actividades propuestas en el aula y fuera de ella, tanto escolares como investigativas en ciencias naturales, matemáticas, ingenierías y otras como la medicina.

Desde el análisis hecho con la ID, se ratifica el carácter dinámico del modelo y su condición de ser sistémico y flexible.

La metodología definida en el modelo posibilita que pueda ser visto como una metodología de investigación, porque garantiza las fases de planeación, diseño de actividades, experimentación y evaluación (confrontación de análisis a priori y a posteriori), con el fin de retroalimentar el proceso investigativo. 
La naturaleza del modelo corresponde a una práctica social normada, determinada en las relaciones que se establecen entre los nodos de planeación y reconocimiento del sujeto, las cuales reconocen a las dimensiones y la formación integral de los sujetos como dinamizadores del MRPPI.

\section{Recomendación}

A través del análisis de las prácticas sociales inmersas en la naturaleza del MRPPI, sería importante que en estas se estudiara la modelación y los usos del conocimiento como aportes socioculturales.

\section{Referencias bibliográficas}

Artigue, M.; Douady, R.; Moreno, L. y Gómez, P. (ed.) (1995). La enseñanza de los principios del cálculo: problemas epistemológicos, cognitivos y didácticos. Ingeniería didáctica en Educación Matemática (pp. 97-140). México: Una Empresa Docente y Grupo Editorial Iberoamérica.

Cantoral, R.; Farfán, R.M.; Lezama, J. y Martínez, G. (2006). Socioepistemología y representación: algunos ejemplos. Relime, número especial, 83-102.

Douady, R. (1995). Nacimiento y desarrollo de la didáctica de las matemáticas en Francia: rol de los IREM en la ingeniería didáctica en educación matemática. Bogotá: Grupo Editorial Iberoamérica.

Farfán, M. (1997). Ingeniería didáctica. Un estudio de la variación y el cambio. México: Grupo Editorial Iberoamérica.

Jessup, C.M. (1998). Resolución de Problemas y Enseñanza de las Ciencias Naturales. Tecné, Episteme y Didaxis: TED, 3, 41-52.

Jessup, C.M. (2004). Resolución de Problemas en la enseñanza de la fisiología. Proyecto de investigación Año sabático. Universidad Pedagógica Nacional

Jessup, C.M. y Pulido, R. (1998). Los Estudios de Calidad de Vida: Alternatividad y Educación Basada en la Investigación. Tecné, Episteme y Didaxis: TED, 4, 111 1-124.

Jessup, M., Pulido, R. (2001). La Resolución de Problemas y la Educación en Ciencias Naturales. Geografías e Imaginarios. (Colombia). ISBN: 958-9097-60-X ed: (pp. 298-311).

Jessup, M. (2000). Evaluación Metodológica de Investigación interacción para el Desarrollo Educativo. Colección Cuadernos de Trabajo 1. Bogotá: Universidad Pedagógica Nacional.

Polya, G. (1979). Cómo plantear y resolver problemas. México: Ed. Trillas.

Pulido, R. (2007). La didáctica de la microbiología centrada en la resolución de problemas en una perspectiva de investigación. Tesis doctoral. Bogotá: Universidad Pedagógica Nacional.

Ramírez, E. (2012). Enseñanza de la función derivada con el uso de infinitesimales como alternativa para reducir los conflictos semióticos de los estudiantes. Doctorado Interinstitucional entre las universidades Valle, Pedagógica Nacional y Francisco José de Caldas. Tesis doctoral. Sin publicar. 


\section{Anexo 1}

Instrumento para la agenda de trabajo del modelo de resolución de problemas en una perspectiva de investigación (MRPPI)

Grupo Probleduciencias

\begin{tabular}{|c|c|c|c|c|c|c|c|c|c|c|c|c|c|c|}
\hline \multirow[b]{2}{*}{ 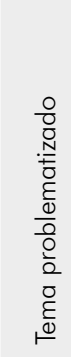 } & \multicolumn{3}{|c|}{ Actividades } & \multicolumn{3}{|c|}{ Contenidos } & \multicolumn{3}{|c|}{$\begin{array}{l}\text { Metas propuestas en la } \\
\text { perspectiva de desarrollo } \\
\text { humano integral }\end{array}$} & \multicolumn{3}{|c|}{$\frac{\text { Evaluación de metas }}{\text { Indicadores }}$} & \multicolumn{2}{|c|}{$\begin{array}{l}\text { Tiempo de } \\
\text { realización }\end{array}$} \\
\hline & 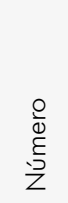 & 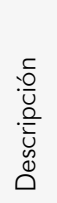 & $\begin{array}{l}\frac{\mathscr{o}}{0} \\
\frac{\partial}{0} \\
\frac{0}{0} \\
\underline{U} \\
0 \\
0\end{array}$ & 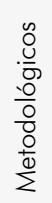 & 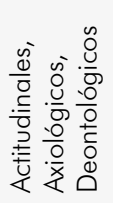 & 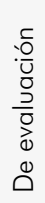 & $\begin{array}{l}\frac{0}{0} \\
\frac{D}{U} \\
\frac{0}{0} \\
\frac{0}{\underline{D}}\end{array}$ & $\begin{array}{l}0 \\
0 \\
\frac{0}{5} \\
\frac{0}{0} \\
\frac{0}{0} \\
\frac{0}{0} \\
\frac{10}{2}\end{array}$ & 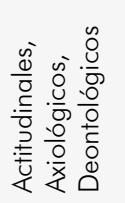 & 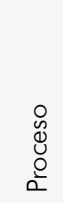 & $\begin{array}{l}\frac{0}{0} \\
\frac{0}{5} \\
\frac{0}{0} \\
\mathscr{d} \\
\alpha\end{array}$ & 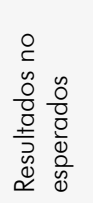 & 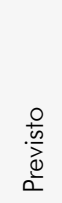 & $\begin{array}{l}\overline{\mathscr{0}} \\
\stackrel{\mathscr{\alpha}}{ }\end{array}$ \\
\hline & 1 & & & & & & & & & & & & & \\
\hline & 2 & & & & & & & & & & & & & \\
\hline & 3 & & & & & & & & & & & & & \\
\hline & 4 & & & & & & & & & & & & & \\
\hline & 5 & & & & & & & & & & & & & \\
\hline & 6 & & & & & & & & & & & & & \\
\hline & 1 & & & & & & & & & & & & & \\
\hline & 2 & & & & & & & & & & & & & \\
\hline & 3 & & & & & & & & & & & & & \\
\hline & 4 & & & & & & & & & & & & & \\
\hline & 1 & & & & & & & & & & & & & \\
\hline & 2 & & & & & & & & & & & & & \\
\hline & & & & & & & & & & & & & & \\
\hline Otros & & & & & & & & & & & & & & \\
\hline & & & & & & & & & & & & & & \\
\hline & & & & & & & & & & & & & & \\
\hline & & & & & & & & & & & & & & \\
\hline & & & & & & & & & & & & & & \\
\hline
\end{tabular}

Autoría. Jessup, Margie. Coordnadora grpo de investigación Problmeduciencias, 2007 